\title{
Hair growth by means of sparse volumetric modeling and advection
}

\author{
Ashraf Ghoniem and Ken Museth \\ DreamWorks Animation
}

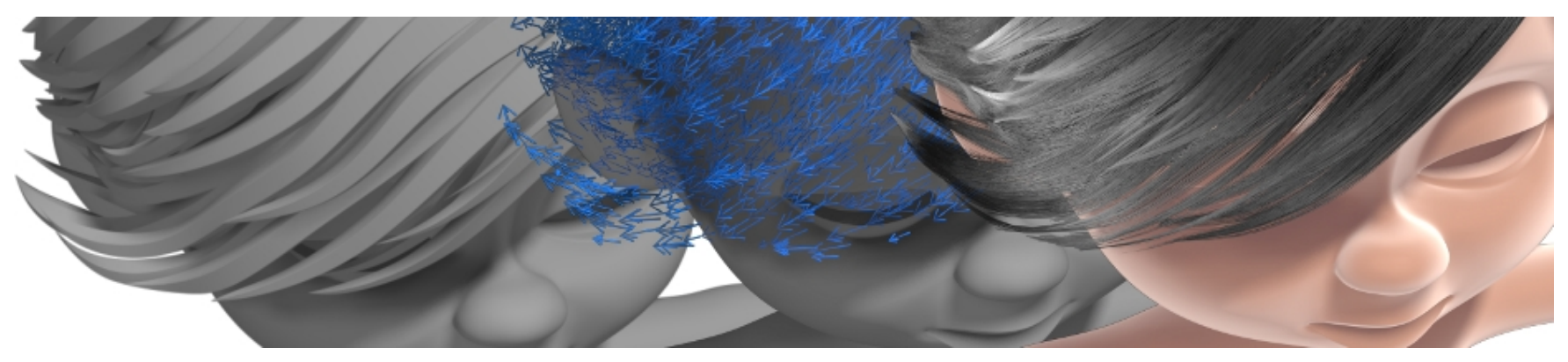

Figure 1: Example showcasing our volumetric approach to hair growth. Left: polygonal model of scalp and hair serving as input to our method. Middle: Sparse volumetric velocity field shown as blue arrows that represent the flow of hair. Right: Final hair curves derived as streamlines from the sparse velocity field.

\begin{abstract}
Summary: We present a fast and flexible technique for creating hair styles directly from modeled geometry by advecting streamlines in sparse high-resolution velocity fields derived from surfaces or curves. Typically artists model hair styles as polygonal surfaces or curves representing sparse guide hairs. From this we automatically create a sparse, volumetric vector field with direction and magnitude derived from the tangents of the input geometry or curves. Additional hair styling is achieved by modifying this vector field, and curves representing the final hair model are computed as streamlines. This volumetric approach represents a significant paradigm shift from traditional curve-based hair modeling in that it allows artists to intuitively create and modify realistic hair styles directly from geometric surface models. Our technique also lends itself well to up-sampling of spares guide hairs without the need to employ explicit curve-based interpolation schemes.
\end{abstract}

Surface Modeling of Hair: Typically the input to our hair grooming system is a collection of polygon (or NURBS) models for the scalp as well as surfaces of hair clumps. Thus, hair is effectively represented by proxy geometry rather than individual curves, which allows artists to work with high-level abstractions like silhouettes and surfaces using existing mesh editing tools. In preparation for the voxelization, the modeled surfaces are closed by procedurally polygonizing unshared edges.

While the idea to generate hair curves from geometric surfaces is certainly not new we believe to be the first to propose a purely volumetric approach that offers interesting new possibilities in terms of artistic control. Notably [Yuksel et al. 2009] proposed a conceptually similar approach except they derived the hair curves by interpolation within polygonal cross-sections of the input meshes. Instead we convert the polygons into a volumetric representation of the tangent flow extended into the interior of the polygon model.

Curve Modeling of Hair: Another common challenge encountered in hair modeling and rendering, is the problem of upsamling high-resolution hair curves from a sparse set of guide hairs. The common approach is to employ various interpolation schemes that operate on a sub-set of the guide hairs to generate new interpolated hair curves. Instead we propose rasterizing the tangent directions of the guide curves into a sparse velocity field. This field can then be combined, using standard volumetric compositing operations, with other flow fields e.g. generated from other hair styles represented either by polygonal surfaces or guide hairs. The resulting flow field is then used to generate new curves defined as streamlines.

Volume Generation and Styling: The interior of the closed polygonal surfaces, or a user-defined neighborhood of the guide hairs, are voxelized into a sparse volumetric representation utilizing a novel compact data structure that allows for high resolutions with small memory footprints [Museth 2013]. Next, a flow field for the hair is created by transferring the tangents of the surface meshes or curves into the voxel grid. This is effectively facilitated by closest point transformations derived directly from the level set representations of the surfaces or curves. The high resolution of these sparse flow fields allows us to capture fine details of the geometric model or curves. Additional styling is then achieved by manipulating and editing the flow volume, e.g. by adding directional fields, procedural vector noise, and other volumetric compositing tools developed at our studio. We will demonstrate these techniques for vector field manipulations to groom production hair models.

Hair Growth by Advection: Finally, curves representing the individual hairs are generated by scattering root points on the scalp model (hair follicle), and advecting streamlines in the flow volume. This approach creates naturalistic parting and growth patterns, e.g. cowlicks. These streamlines can then be used for both hair simulation and rendering. Resolution of the final model is easily controlled by integrating a variable number of streamlines without changing any of the upstream modeling operations. In the accompanying video we show examples hereof for straight, wavy, and curly hair. This grooming technique is an important part of a larger toolbox for hair modeling, grooming and simulation tools, with debut use on DreamWorks Animation's Turbo.

\section{References}

Museth, K. 2013. VDB: High-resolution sparse volumes with dynamic topology. ACM Trans. Graph. 32, 3 (May), 23.

Yuksel, C., Schaefer, S., AND Keyser, J. 2009. Hair meshes. ACM Trans. Graph. 28, 5 (Dec.), 166:1-166:7. 